Karstenia 40: 39-41, 2000

\title{
Myxomycetes of Turkey
}

\author{
C. CEMERGÜL and BASARAN DÜLGER
}

ERGÜL, C.C. \& DÜLGER, B. 2000: Myxomycetes of Turkey. - Karstenia 40: 39-41. Helsinki. ISSN 0453-3402.

This paper contains a complete list of Myxomycetes of Turkey. The checklist, which is mainly based on literature records, includes 102 taxa, belonging to 31 genera.

Key words: Turkey, Myxomycetes, checklist

C.Cem Ergül \& Basaran Dülger, Department of Biology, University of Uludag, Faculty of Science \& Letter, 16059, Bursa, Turkey

\section{Introduction}

Work on Turkish myxomycete flora is very recent. The first myxomycete records are in works on macrofungi (Lohwag 1957, 1964). Detailed work by Finnish scientists followed after a gap of twenty years (Härkönen \& Uotila 1983, Uotila \& Kurtto 1984, Härkönen 1987). The first specific accounts by Turkish workers began later (Gücin \& Öner 1986). In particular, a PhD thesis on the myxomycetes of the Southern Marmara region increased the number of known Turkish species (Ergül 1993). In the following years there has been an important increase in records (Ergül \& Gücin 1993, 1994, 1995, 1996; Ergül 1997; Ergül \& Dülger 1999 a-c; Ergül 1999; Gücin \& Ergül 1995; Gün 1995). A major checklist of the myxomycetes of the Mediterranean region includes Turkish records (Lado 1994). Extending the study of myxomycetes from Marmara, the Aegean coast and the western Black Sea region to the whole country will reveal the richness of the Turkish myxomycete flora. Turkey, is at the intersection of four phytogeographical regions (Mediterranean, Euro-Siberian, Mesopotamian and Irano-Turanian) and has a rich natural vegetation with different macro-microclimates, and is thus an ideal study area.

\section{Methods}

The checklist is based on literature records of myxomycetes collected in Turkey. The generic treatment follows Martin et al. (1983). Species are those accepted by Martin \& Alexopoulos (1969) and Nannenga Bremekamp (1991).

\section{The checklist}

Division: Gymnomycota

Subdivision: Plasmodiogymnomycota

Classis: Myxomycetes

Subclassis: Ceratiomyxomycetidae

Order: Ceratiomyxales

Family: Ceratiomyxaceae

Ceratiomyxa fruticulosa (O.F. Muell.) T. Macbr. Härkönen 1987; Ergül 1993; Gün 1995

Subclassis: Myxogasteromycetiadae

Order: Liceales

Family: Liceaceae

Licea castanea G. Lister - Ergül 1993

L. denudescens Keller \& Brooks - Härkönen \& Uotila 1983

L. kleistobolus Martin - Härkönen \& Uotila 1983;

Härkönen 1987

L. minima Fries - Ergül 1993

L. operculata (Wingate) Martin - Ergül 1993

L. parasitica (Zukal) Martin - Ergül 1993

L. pedicellata (H.C. Gilbert) H.C. Gilbert - Ergül 1993

L. punctiformis Martin - Ergül 1993

L. pusilla Schrad. - Ergül 1993

L. pusilla var. pygmaea Meylan - Ergül 1993; Ergül \& Dülger 1999

L. tenera Jahn - Ergül 1993

Family: Cribrariaceae

Cribraria aurantiaca Schrad. - Ergül 1998

C. cancellata (Batsch) Nann.-Bremek - Ergül 1993;

Ergül \& Gücin 1993; Ergül \& Dülger 1999a

C. minutissima Schw. - Ergül 1998 
C. violacea Rex - Härkönen \& Uotila 1983; Ergül 1993; Gün 1995; Ergül \& Dülger 1999a

Family: Enteridiaceae

Enteridium splendens (Morgan) Macbr. - Gücin \& Ergül 1995; Gün 1995

Lycogala epidendrum (L.) Fries - Härkönen 1987; Ergül 1993; Gün 1995; Ergül \& Dülger 1999a

L. flavofuscum (Ehrenb.) Rostaf. - Ergül 1993

Order: Echinosteliales

Family: Echinosteliaceae

Echinostelium arboreum Keller \& Brooks - Ergül 1993

E. coelocephalum Brooks \& Keller - Ergül 1993; Gün 1995

E. colliculosum Whitney \& Keller - Härkönen \& Uotila 1983; Ergül 1993; Gün 1995

E. corynophorum Whitney - Gün 1995

E. cribrarioides Alexop. - Ergül 1993

E. elachiston Alexop. - Härkönen 1987

E. fragile Nann.-Brem. - Ergül 1993; Gün 1995

E. minutum de Bary - Härkönen \& Uotila 1983; Härkönen 1987; Ergül 1993; Gün 1995, Ergül \& Dülger 1999a

Family: Clastodermataceae

Clastoderma debaryanum Blytt - Ergül 1993

C. pachypus Nann.-Brem. - Ergül 1993

Order: Trichiales

Family: Arcyriaceae

Arcyria cinerea (Bull.) Pers. - Härkönen \& Uotila 1983; Härkönen 1987; Ergül 1993; Gün 1995; Ergül \& Dülger 1999a

A. denudata (L.) Wettst. - Härkönen 1987; Ergül 1993; Gün 1995

A. incarnata (Pers.) Pers. - Ergül 1993; Gün 1995; Ergül \& Dülger 1999a

A. insignis Kalchbr. \& Cooke - Ergül 1993

A. minuta Buchet - Ergül \& Dülger 1999b

A. nutans (Bull.) Grev. - Ergül 1993; Ergül \& Dülger 1999a

A. pomiformis (Leers) Rost. - Härkönen \& Uotila 1983; Härkönen 1987; Ergül 1993; Gün 1995

A. versicolor Phill. - Härkönen 1987; Gün 1995

Perichaena chrysosperma (Currey) Lister - Ergül 1993; Gün 1995; Ergül \& Dülger 1999a

P. corticalis (Batsch) Rostaf. - Härkönen \& Uotila 1983; Härkönen 1987; Ergül 1993; Gün 1995

P. vermicularis (Schw.) Rost. - Ergül 1993; Gün 1995

Family: Dianemaceae

Calomyxa metallica (Berk.) Nieuwl. - Ergül 1993

Family: Trichiaceae

Trichia alpina (R.E.Fries) Meylan - Härkönen 1987

T. botrytis (J.F.Gmel.) Pers. - Ergül 1993

T. contorta (Ditmar) Rost. - Härkönen \& Uotila 1983

T. contorta var. karstenii (Rostaf.) Ing - Ergül \&

Gücin 1995

T. decipiens (Pers.) Macbr. - Härkönen 1987; Ergül 1993

T. favoginea (Batsch) Pers. - Härkönen 1987; Ergül 1993

T. lutescens (Lister) Lister - Gücin \& Öner 1986

T. varia (Pers.) Pers. - Gücin \& Öner 1986; Ergül 1993; Gün 1995

Hemitrichia calyculata (Speg.) Farr. - Ergül 1999

H. clavata (Pers.) Rostaf. - Härkönen 1987
Metatrichia vesparium (Batsch) Nann.-Bremek. Ergül \& Gücin 1993

Order : Physarales

Family: Physaraceae

Badhamia foliicola Lister - Härkönen \& Uotila

1983; Ergül 1993; Gün 1995; Ergül \& Dülger 1999a

B. macrocarpa (Ces.) Rostaf. - Gücin \& Öner 1986; Ergül 1993

B. nitens Berk - Ergül 1993

B. panicea (Fries) Rostaf. - Härkönen \& Uotila 1983

B. versicolor Lister - Härkönen \& Uotila 1983

B. viridescens Meylan - Ergül 1992

Badhamiopsis ainoae (Yamash.) Brooks \& Keller Ergül 1993; Gün 1995

Fuligo septica (L.) Wiggers - Ergül \& Gücin 1994

Leocarpus fragilis (Dicks.) Rostaf. - Gün 1995; Ergül

\& Dülger 1999a

Physarum auriscalpium Cooke - Härkönen \& Uotila

1983; Härkönen 1987

P. bitectum G. Lister - Härkönen \& Uotila 1983

P. cinereum (Batsch) Pers. - Härkönen 1987; Ergül 1993

P. compressum Alb. \& Schw. - Ergül 1993

P. contextum (Pers.) Pers. - Härkönen \& Uotila 1983

P. decipiens Curt. - Ergül 1993

P. leucophaeum Fries - Ergül 1993; Gün 1995

P. nutans Pers. - Ergül 1993; Gün 1995; Ergül \& Dülger 1999 a

P. oblatum Macbr. - Ergül 1993

P. pusillum (Berk. \& Curt.) G. Lister - Ergül 1997; Ergül \& Dülger 1999a

P. viride (Bull.) Pers. - Ergül 1993; Ergül \& Dülger 1999a

Family: Didymiaceae

Diderma chondrioderma (de Bary \& Rost.) G. Lister

- Ergül 1992; Ergül \& Dülger 1999a

D. hemisphaericum (Bull.) Hornem. - Ergül 1993

Didymium difforme (Pers.) S.F.Gray - Ergül 1993; Gün 1995

D. floccosum Martin, Thind \& Rehill - Ergül \& Gücin 1996; Ergül \& Dülger 1999a

D. minus (Lister) Morgan - Ergül \& Gücin 1996

D. quitense (Pat.) Torrend - Härkönen \& Uotila 1983

D. squamulosum (Alb. \& Schw.) Fries - Härkönen \&

Uotila 1983

Mucilago crustacea Wiggers - Ergül 1999

Subclassis: Stemonitomycetidae

Order : Stemotiales

Family: Stemonitaceae

Amaurochaeta atra (Alb.\&Schw.) Rost. - Sümer 1982 Comatricha dictyospora Celak. - Ergül 1993; Gün 1995

C. elegans (Racib.) G.Lister - Härkönen \& Uotila 1983; Ergül 1993

C. ellae Härkönen - Ergül 1993; Gün 1995

C. laxa Rost. - Härkönen \& Uotila 1983; Ergül 1993;

Gün 1995; Ergül \& Dülger 1999a

C. lurida Lister - Ergül 1993

C. nigra (Pers.) Schroet. - Härkönen \& Uotila 1983; Ergül 1993; Gün 1995; Ergül \& Dülger 1999a

Diachea leucopodia (Bull.) Rost. - Härkönen \& Uotila 1983

Enerthenema papillatum (Pers.) Rostaf. - Härkönen \& Uotila 1983; Ergül 1993; Gün 1995 
Lamproderma arcyrioides ( Sommerf.) Rostaf. Härkönen 1987

Macbrideola cornea (G.Lister \& Cran) Alexop. Härkönen \& Uotila 1983; Härkönen 1987; Ergül 1993; Gün 1995

M. decapillata H.C.Gilbert - Ergül 1993

M. synsporos (Alexop.) Alexop. - Ergül 1993; Gün 1995

Paradiacheopsis fimbriata (G.Lister \& Cran) Hertel - Härkönen \& Uotila 1983; Härkönen 1987; Gün 1995 Stemonitis flavogenita Jahn - Härkönen \& Uotila 1983

S. fusca Roth - Härkönen \& Uotila 1983; Ergül \& Dülger 1999a

S nigrescens Rex - Ergül 1993; Gün 1995

S. splendens Rostaf. - Uotila \& Kurtto 1983

Stemonitopsis hyperopta (Meylan) Nann.-Bremek. Ergül 1993

S. typhina (F.H.Wigg.) Nann.-Bremek. - Härkönen 1987

Symphytocarpus flaccidus (Lister) Ing \& Nann.Bremek. - Ergül \& Dülger 1999c

\section{Results}

In the past, very few studies have been made on myxomycetes of Turkey. Now the myxomycetes in Turkey number 102 taxa belonging to 31 genera. Since most myxomycetes are thought to be cosmopolitan, further explorations will undoubtly increase the knowledge of myxomycetes of Turkey.

\section{References}

Ergül, C. C. 1992: Two new Myxomycetes taxa for Turkish mycoflora. - Istanbul Univ. Fen Fak. Biyoloji Der. 56: 57-61.

Ergül, C. C. 1993: The taxonomic investigations of the Myxomycetes which have been collected on the Marmara region of Anatolian division. - Ph. Doctoral Thesis (unpublished), Uludag University, Bursa. 173 pp.

Ergül, C. C. 1997: A new record of Myxomycete for Turkish mycoflora (= Physarum pussilum) Berkeley \& Curtis) G.Lister). - Al-Azhar University Bulletin of Science, 409-414.

Ergül, C. C. 1998: Two new records of Myxomycetes taxa for Turkish mycoflora. - Sci. Int. (Lahore), 10 (2): $173-176$.

Ergül, C. C. 1999: Two new records of Myxomycetes for the Turkish mycoflora. - ICSEM-3, Abstract Volume p. 53

Ergül, C. C. \& Dülger, B. 1999a: The Myxomycetes of Görükle campus area (Bursa). - Ot Sistematik Dergisi (in press).
Ergül, C. C. \& Dülger, B. 1999b: A new Myxomycetes record for the Turkish mycoflora. - Tr. J. Botany (in press).

Ergül, C. C. \& Dülger, B. 1999c: Türkiye Miksomiset florasi için yeni bir takson: Symphytocarpus B. Ing \& Nann.-Brem. - Ot Sistematik Dergisi.

Ergül, C. C. \& Gücin, F. 1993: Türkiye için yeni iki Myxomycetes taksonu. - Tr. J. Botany 17: 267-271.

Ergül, C. C. \& Gücin, F. 1994: Türkiye Myxomyceteleri için yeni bir kayit (Fuligo septica (L.) Wiggers). XII. Ulusal Biyoloji Kongresi, Cilt II, Bot. Sect., Edirne, 157-159.

Ergül, C. C. \& Gücin, F. 1995: Türkiye için yeni bir Myxomycet taksonu: Hemitrichia Rost. - Tr. J. Botany 19: $165-166$.

Ergül, C. C. \& Gücin, F. 1996: Two new records of Myxomycetes taxa for Turkish mycoflora. - Plant Life in Soutwest and Central Asia, Ege Univ. Pres. 2: 432-439.

Gücin, F. \& Ergül, C.C. 1995: A new Myxomycetes genus (Enteridium) record for the Turkish mycoflora. Tr. J. Botany 19: 565-566.

Gücin, F. \& Öner, M. 1986: Taxonomic observations on some Turkish Myxomycetes species. - Journal of Firat University 1(1): 19-28.

Gün, Z. 1995: Uludag'in farkli vejetasyon zonlarindaki agaçlarin kabuklarindan izole edilen Myxomycetes türleri üzerinde taksonomik arastirma. - Master Thesis (unpublished), Uludag Üniversity, Bursa. 120 pp.

Härkönen, M. 1987 (1988): Some addition to the knowledge of Turkish Myxomycetes. - Karstenia 27: $1-7$.

Härkönen, M. \& Uotila, P. 1983: Turkish Myxomycetes developed in moist chamber cultures. - Karstenia 23: $1-9$.

Lado, C. 1994: A checklist of Myxomycetes of the Mediterranean Countries. - Mycotaxon LII (1): 117 185.

Lohwag, K. 1957: Türkiye'nin Mantar Florasi hakkinda arastirma. - I.Ü.O.F. Der. Ser. A. 7 (1): 129-137.

Lohwag, K. 1964: Belgrad ormanindan mikolojik notlar. - I.Ü.O.F. Der. Ser. B. 14 (2): 128-135.

Martin, G. W. \& Alexopoulos, C J. 1969: The Myxomycetes. - Iowa City. $560 \mathrm{pp}$.

Martin, G. W., Alexopoulos, C. J. \& Farr, M. L. 1983: The genera of Myxomycetes. - Iowa City. 561 pp.

Nannenga-Bremekamp, N. E. 1991: A guide to temperate Myxomycetes. - Biopress Limited, Bristol. 409 pp.

Sümer, S. 1982: Bati Karadeniz bölgesi, özellikle Bolu çevresinde bulunan odun tahripçisi mantarlar. - Istanbul Orman Fakültesi Yayinlari 321: 1-194.

Uotila, P. \& Kurtto, A. 1984: Helsingin yliopiston kasvitieteen laitoksen Turkin retki 1983. - Helsingin yliop. kasvit. lait. monisteita 90: 1-63. 